for children/young people and adults, complementary therapy as well as individualised carers' support and access to a menu of therapeutic groups.

There are age-related outcome-based evaluations in place to measure the effectiveness of the group. All service users are encouraged to feedback any issues that would improve their experience of the group.

There is no similar group in Essex to meet the support needs of this group of people. In essence the Clan Club is a warm, friendly group that values individuality, promotes empowerment, but most importantly provides a focus for families to meet.

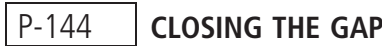

Manisha Cook, Caroline Whelldon. Hospice of St Francis, Berkhamsted, UK

\subsection{6/bmjspcare-2016-001245.167}

Background A retrospective study into the experiences of pulmonary fibrosis patients and carers identified holistic assessment and carer support as the main unmet needs of this client group. Pulmonary fibrosis is generally a rapidly progressive disease, causing great physical and psychological distress to those affected. The disease trajectory of Pulmonary fibrosis differs from Chronic Obstructive Pulmonary Disease (COPD) but often they are treated together in exercise and support groups.

With the aim of addressing the lack of specific pulmonary fibrosis support and to meet patient and carer needs a hospice based pulmonary fibrosis support group was established.

Method A twice monthly group was established within the Hospice of St Francis, led by a specialist palliative care physiotherapist with support from a palliative care nurse specialist. The sessions include a patient-led exercise programme and conclude with relaxation. Regular speakers join the group covering topics from future care planning, cognitive behavioural therapy, diet, fatigue management and social services.

Each patient completes a holistic needs assessment and we are commencing the use of Well-being star outcome measure tool to evaluate effectiveness. Carers are invited to complete a carer's support needs assessment tool (CSNAT).

Conclusion and future goals The group is still in its infancy. The aim is for sessions to be patient led with a nominated patient or carer as chairperson, treasurer etc.

We are engaging with local respiratory groups, lung clinics and Pulmonary Fibrosis charities to reach out to as many patients and carers within Hertfordshire and bordering counties and expand/ establish the group further. The most effective form of advertising has been through social media.

Carer focus is developing with plans to host education and peer support sessions. Our aim is to arm carers with knowledge, build confidence in regards to symptom management and future care planning. HOSPICE-PALLIATIVE CARE AMONG TERMINAL CANCER PATIENTS IN KOREA, 2008-2014

1,2So Jung Park, 1,3Yoon Jung Chang, 'Ju Yeon Bak, 'Eun Jung Park, ' ${ }^{1}$ Hyun Jung Jho, ${ }^{1}$ Jin Young Choi, 'Eun Mi Ahn, ${ }^{1,3}$ Yeol Kim. ${ }^{1}$ Palliative Care Clinic, National Cancer Centre, Republic of Korea; ${ }^{2}$ Department of Family Medicine, Graduate School of Yonsei University, Seoul, Republic of Korea; ${ }^{3}$ Department of Cancer Control and Policy, Graduate School of Cancer Science and Policy, Republic of Korea

\subsection{6/bmjspcare-2016-001245.168}

Background Utilisation of hospice-palliative care (HPC) has increased steadily; however, little is known about trend or pattern of this use in Korea. We aimed to describe an overview of nationwide statistics on the utilisation of HPC service of terminal cancer patients in Korea.

Methods Data were collected through Korean Terminal Cancer Patient Information System from Hospice-Palliative Care Units (PCU) designated by Korea's Ministry of Health and Welfare from 2008 to 2014. Descriptive statistics were used for the analysis. Trends were examined by annual percentage change and Cochran-Armitage test.

Results 56,433 patients used PCU for seven years. In 2008, only 19 hospitals (total 282 beds) were designated as PCU for terminal cancer patients by Korean government. With gradual increases, there were 57 designated PCU (total 950 beds) in 2014. The utilisation rate of PCU by terminal cancer patients increased from $7.3 \%$ of all cancer death in 2008 to $13.8 \%$ in 2014 . The patients' mean age was $67.1 \pm 12.9$ years, and $57.3 \%$ were male. Lung cancer patients made up the largest percentage of PCU admission. Increasing trends were observed in patient's awareness of terminal status from $67.3 \%$ in 2010 to $76.1 \%$ in 2014 . Average length of stay was $23.3 \pm 27.0$ days and $73.9 \%$ of discharged patients faced death in PCU.

Conclusions The number of terminal cancer patients received hospice care has steadily increased over the past several years in Korea. It is necessary to develop a variety of services that enhance the quality of end of life care by monitoring of hospice utilisation.

\section{P-146 SHARED CARE LIVER PROJECT}

Virginia Campbell, Sharon Quinn. St Luke's Hospice, Basildon, UK

\subsection{6/bmispcare-2016-001245.169}

There is growing recognition that people with advanced liver disease (ALD) have limited access to palliative care, despite a high symptom, psychological and social burden. Many have little opportunity to do end-of-life planning and some are referred to palliative care services as late as two days before death

Early hospice referral for patients with ALD and their carers seems at best a national aspiration rather than standard best practice. 